of difference between cancer and SSc diagnosis. No association was found between neoplasia and certain antibodies. We recommend further studies to evaluate the relationship between SSc and cancer.

Disclosure of Interests: None declared

DOI: 10.1136/annrheumdis-2020-eular.4827

\section{THU0636-HPR REMS TECHNOLOGY APPLIED TO RHEUMATIC DISEASE}

M. D. Tomai Pitinca ${ }^{1}$, C. Caffarelli ${ }^{2}$, S. Gonnelli ${ }^{2}{ }^{1}$ National Research Council, Institute of Clinical Physiology, Lecce, Italy; ${ }^{2}$ Department of Medicine, Surgery and Neuroscience. University of Siena, Siena, Italy

Background: Many Rheumatic disease such as rheumatoid arthritis, juvenile idiopathic arthritis, psoriatic arthritis, ankylosing spondylitis, systemic lupus erythematosus, dermatomyositis/polymyositis and vasculitis are characterised by osteoporosis and fragility fractures. Inflammatory cytokines, steroid treatment, immobilization and reduced physical activity due to joint pain and muscle weakness are considered the major risk factors for the development of low bone mineral density in these diseases. Many evidences have highlighted the role of pro-inflammatory cytokines (TNF-a, IL-1, IL-6, IL-7, IL17) in bone homeostasis regulation.Chronic inflammation is often characterized by an imbalance between bone formation and resorption, with a clear prevalence of osteoclastogenesis which is a strong determinant in rheumatic diseases bone loss.

Objectives: The aim of this study is to evaluate the REMS (Radiofrequency Echographic Multi-Spectrometry) technology in rheumatologic patients, compared to DEXA currently recognised as the gold standard for the evaluation of bone mineral density.

Methods: Twenty female patients (mean age $60.6 \pm 14.41$ years) with different rheumatologic diseases were considered. Each patient underwent a lumbar spine and hip examination performed by DEXA and REMS technology. In particular, after a quality control to assess that both the exams were performed correctly, 18 lumbar and 20 femoral exams (DEXA vs REMS) were compared.

Results: As Expected the exams performed show a good diagnostic match ( $>60 \%$ LS and $>85 \%$ FEMORE). The tests that didn't show diagnostic concordance were those affected by arthrosis processes (greater on the Spine). The REMS T-score values were lower than those obtained with the DXA method. Conclusion: These results show how REMS technology can discriminate patients with osteoporosis as much as DEXA technology. The REMS technology can be a diagnostic option especially in patients with rheumatologic diseases that cause alterations in the spine reducing the diagnostic sensitivity of DXA technology.

Disclosure of Interests: None declared

DOI: 10.1136/annrheumdis-2020-eular.6500

\section{THU0637-HPR THE ASSOCIATION BETWEEN THE RECALL PERIOD AND THE AMOUNT OF INFORMATION ABOUT REPORTED ADVERSE DRUG REACTIONS BY PATIENTS USING BIOLOGICALS}

A. Laurijssen ${ }^{1}$, J. Van Lintt $^{2}$, B. Van den Bemt ${ }^{1,3}$, L. Beijer ${ }^{1,3}$, N. Jessurun ${ }^{2}$ ${ }^{1}$ Radboud University, Nijmegen, Netherlands; ${ }^{2}$ Pharmacovigilance centre Lareb, 's Hertogenbosch, Netherlands; ${ }^{3}$ Sint Maartenskliniek, Nijmegen, Netherlands

Background: In order to monitor the safety of medicines pharmacovigilance, it is important that patients report their adverse drug reactions (ADRs). Theoretically, the quality of the reported information might be affected by the elapsed time between the onset of the ADR and the moment of reporting. Real-life evidence demonstrating a negative relationship between this recall period and the quality of reported ADRs is however lacking.

Objectives: To assess the effect of recall period on the amount of information that patients report about their ADR (information density) in patients using a biologic for an immune-mediated inflammatory disease (IMID).

Methods: The Dutch Biologic Monitor is a multi-center cohort ADR monitoring system collecting data on reported ADRs by patients using a biologic for an IMID. Per patient, every first unique reported ADR between 1 February 2017 and 1 September 2019 was eligible. ADR reports were selected by stratified random sampling based on length of recall period and biologic. The recall period was defined by the number of days between the onset and reporting date of the ADR. The amount of information in an ADR report (information density) was determined based on eleven domains: specification, location, frequency, time-toonset, course, causality, cause or consequence, health care professional (HCP) visits, HCP action, patient action and ADR burden. Information density was calculated by the number of reported domains divided by the number of domains deemed relevant in the ADR report. The association between the information density of the ADR reports and different recall periods was compared using a one-way ANOVA test. One-way ANOVA and independent t-tests were used to assess the impact of gender, age, type biologic and burden of the ADR on the information density of the reported ADRs

Results: Out of 1109 reported ADRs by 531 IMID patients, we included 402 ADR reports of 294 patients (55\%) (see table 1 ). Included reports were equally divided over seven different recall periods: 0-1, 1-2, 2-4, 4-8, 8-12, 12-26 and 26-52 weeks. Results have shown no association between the information density in patient-reported ADRs and the length of recall period $(p=0.805)$ (figure 1). However, the proportion of reported information about HCP visits for the ADR increased with increasing recall period: 0 -1 week (14\%), 1-2 weeks $(24 \%), 2-4$ weeks (34\%), 4-8 weeks (40\%), 8-12 weeks (48\%), 12-26 weeks $(50 \%)$ and 26-52 weeks (46\%).

Table 1. Characteristics of included patients with adverse drug reactions

\begin{tabular}{lc}
\hline Characteristics (N=294) & $\mathbf{N}(\%)$ \\
\hline Gender (female) & $202(69 \%)$ \\
Age (years) (mean \pm SD) & $53 \pm 13$ \\
Smoking & $59(20 \%)$ \\
BMI (kg/m ${ }^{2}$ ) (mean \pm SD) & $25.7 \pm 5.3$ \\
Reported ARDs (mean \pm SD) & $1.4 \pm 0.8$ \\
Indication & \\
Rheumatoid arthritis & $129(44 \%)$ \\
Psoriatic arthritis & $51(17 \%)$ \\
Axial spondyloarthritis & $43(15 \%)$ \\
Crohn's disease & $42(14 \%)$ \\
Other indications & $29(10 \%)$ \\
Biologic & \\
Adalimumab & $97(33 \%)$ \\
Etanercept & $72(24 \%)$ \\
Infliximab & $27(9 \%)$ \\
Tocilizumab & $16(5 \%)$ \\
Secukinumab & $15(5 \%)$ \\
Rituximab & $14(5 \%)$ \\
Other biologics & $53(18 \%)$ \\
\hline
\end{tabular}

Female patients reported more information about their ADR $(p=0.002)$, whereas the patient's age was not associated with information density $(p=0.221)$. Etanercept $(E T A)$ users report significantly more information than adalimumab (ADA) users $(p=0.019)$. The number of patients using other biologics was too low for further analysis. A higher ADR burden tended $(p=0.120)$ to result in more reported ADR information (figure 2).

Conclusion: The length of recall period did not affect the amount of information that patients report about their $\operatorname{ADR}(\mathrm{s})$. The recall period was longer for patients reporting information about their HCP visit. Furthermore, female patients tend to report more information about their ADR than male patients and ETA-users tend to report more than ADA-users.

Figure 1: No effect of length of recall period on information density in patient-reported ADRs

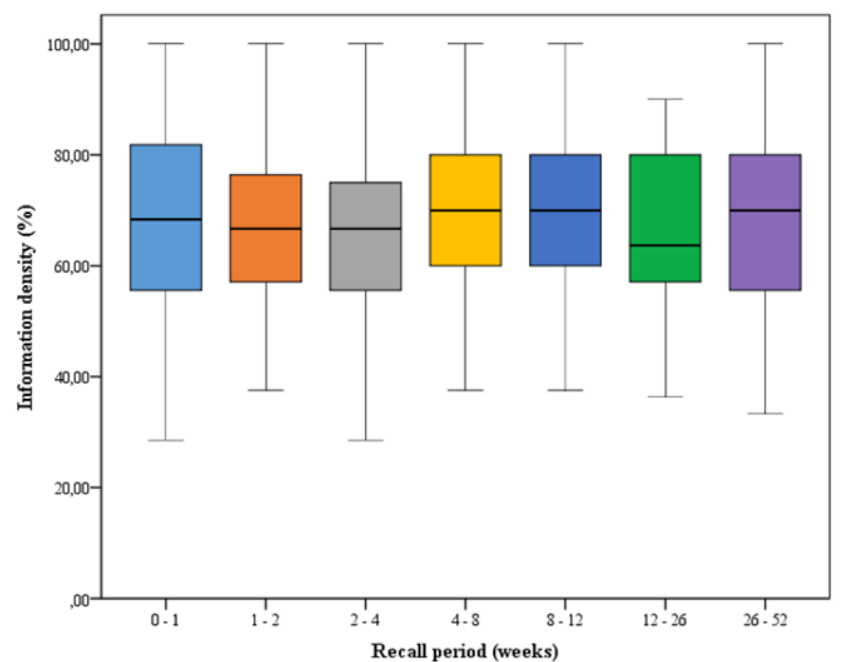




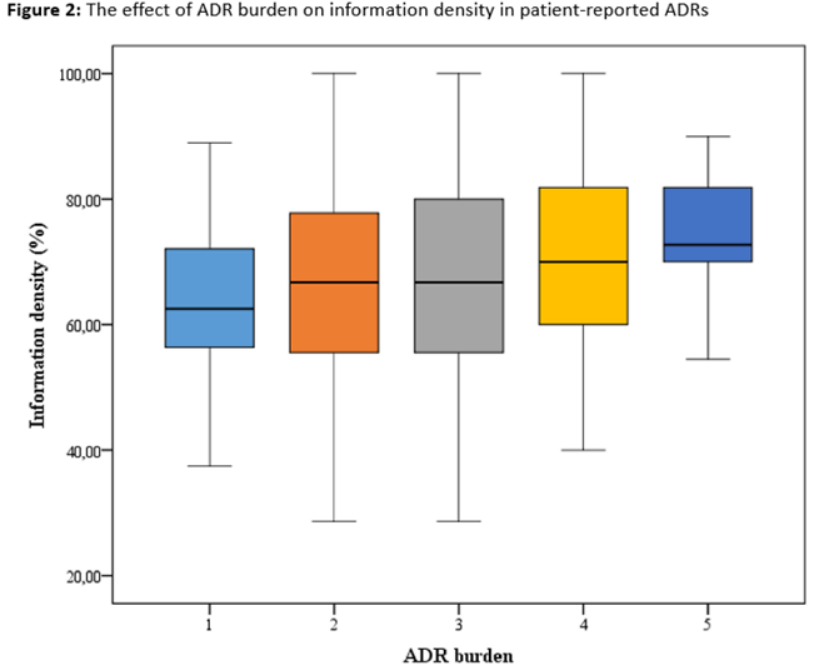

Patients reported their ADR burden on a 5-point scale, ranging from 1 (no burden) to 5 (very high burden)

Disclosure of Interests: Alexandra Laurijssen: None declared, Jette van Lint: None declared, Bart van den Bemt Grant/research support from: UCB, Pfizer and Abbvie, Consultant of: Delivered consultancy work for UCB, Novartis and Pfizer, Speakers bureau: Pfizer, AbbVie, UCB, Biogen and Sandoz., Lilian Beijer: None declared, Naomi Jessurun: None declared

DOI: 10.1136/annrheumdis-2020-eular.4124

\section{THU0638-HPR PHYSICAL ACTIVITY LEVELS OF RADIOGRAPHIC AND NON-RADIOGRAPHIC AXIAL SPONDYLOARTHRITIS PATIENTS}

T. Yuksel-Karsli ${ }^{1}$, D. Bayraktar ${ }^{2}$, D. Ozer Kaya ${ }^{2}$, Ö. Gerçik ${ }^{3}$, S. Gucenmez ${ }^{4}$, H. E. Oz ${ }^{3}$, D. Solmaz ${ }^{3}$, S. Akar ${ }^{3} .{ }^{1}$ Izmir Katip Celebi University, Faculty of Medicine, Department of Internal Medicine, Izmir, Turkey; ${ }^{2}$ Izmir Katip Celebi University, Faculty of Health Sciences, Department of Physiotherapy and Rehabilitation, Izmir, Turkey; ${ }^{3}$ Izmir Katip Celebi University, Faculty of Medicine, Department of Internal Medicine, Division of Rheumatology, Izmir, Turkey; ${ }^{4}$ Izmir Katip Celebi Unviersity, Ataturk Education and Research Hospital, Department of Rheumatology, Izmir, Turkey

Background: It is known that cardiovascular disease risk is increased in chronic inflammatory diseases. Additionally, regular exercise is one of the main components of the management of patients with axial spondyloarthritis (axSpA). However, it was reported that axSpA patients do not meet recommended physical activity levels. It is still unknown, whether the disease subgroups of axSpA play a role in participating in physical activity.

Objectives: To compare the physical activity levels among radiographic, non-radiographic axSpA patients, and healthy controls.

Methods: Thirty-three patients with radiographic axSpA (23 [70\%] male), 33 patients with non-radiographic axSpA $(23[70 \%]$ male) and 33 age and sex matched healthy controls (23 male [70\%]) were included in the study. axSpA patients were assessed regarding to disease activity (Bath Ankylosing Spondylitis Disease Activity Index; BASDAI), functional status (Bath Ankylosing Spondylitis Functional Index; BASFI), spinal mobility (Bath Ankylosing Spondylitis Metrology Index; BASMI). Physical activity level of all subjects was measured by using an accelerometer (Actigraph wGT3X-BT) which was worn on the waist for seven consecutive days.

Results: The groups were similar in terms of physical characteristics (age and body mass index) ( $p>0.05)$. Disease related characteristics (BASMI, BASFI, BASDAI) were comparable between radiographic and non-radiographic axSpA patients $(p>0.05)$. Radiographic axSpA patients showed lesser physical activity compared to non-radiographic axSpA patients and healthy controls $(p<0.05$, Table 1$)$. There is no difference between non-radiographic axSpA patients and healthy controls regarding the physical activity levels $(p>0.05)$.
Table 1. Comparison of Groups

\begin{tabular}{|c|c|c|c|c|}
\hline & $\begin{array}{c}\text { Radiographic } \\
\text { axSpA (n: } 33 \text { ) } \\
\text { Median (IQR } \\
25 / 75)\end{array}$ & $\begin{array}{c}\text { Non-radiographic } \\
\text { axSpA (n: 33) } \\
\text { Median (IQR 25/75) }\end{array}$ & $\begin{array}{l}\text { Healthy Controls } \\
\text { (n:33) } \\
\text { Median (IQR 25/75) }\end{array}$ & $\mathrm{p}$ \\
\hline \multicolumn{5}{|c|}{ Physical Characteristics } \\
\hline Age (years) & $41.0(32.0 / 46.0)$ & $37.0(32.0 / 40.0)$ & $33.0(28.0 / 41.0)$ & $0.093^{*}$ \\
\hline BMI $\left(\mathrm{kg} / \mathrm{m}^{2}\right)$ & $26.0(22.9 / 29.6)$ & $26.3(25.4 / 28.7)$ & $24.8(22.3 / 26.9)$ & $0.064^{*}$ \\
\hline \multicolumn{5}{|c|}{ Disease Related Characteristics } \\
\hline BASMI (score) & $2.1(1.5 / 3.9)$ & $1.5(1.1 / 2.0)$ & NA & $0.051^{\star \star}$ \\
\hline BASFI (score) & $2.4(0.7 / 3.9)$ & $1.2(0.6 / 2.9)$ & NA & $0.267^{\star \star}$ \\
\hline $\begin{array}{l}\text { BASDAI (score) } \\
\text { Physical Activity Lev }\end{array}$ & $3.6(1.6 / 5.8)$ & \multicolumn{2}{|c|}{ Physical Activity Level } & $0.519^{* *}$ \\
\hline Light Physical & 2198.0 & $2576.0^{\#}$ & 2200.0 & $0.015^{*}$ \\
\hline $\begin{array}{l}\text { Activity } \\
\text { (min) }\end{array}$ & $(1377.0 / 2658.0)$ & $(1858.0 / 3690,0)$ & $(1846.0 / 2762.0)$ & \\
\hline Medium Physical & 188.0 & 264.0 & 363.0 & $\mathrm{p}<0.001^{*}$ \\
\hline Activity (min) & $(109.0 / 304.0)$ & $(216.0 / 446.0)$ & $(267.0 / 491.0)^{\# \#}$ & \\
\hline Vigorous Physical & 0.0 & 2.0 & 4.0 & $0.009^{*}$ \\
\hline Activity (min) & $(0.0 / 0.1)$ & $(0.0 / 12.0)$ & $(0.0 / 19.0)^{\# \#}$ & \\
\hline Total Step Count & 42481.0 & 62872.0 & 69710.0 & $\mathrm{p}<0.001^{*}$ \\
\hline (n) & $(33651.0 / 57047.0)$ & $(53075.0 / 80160.0)^{\#}$ & $(59943.0 / 85894.0)^{\# \#}$ & \\
\hline
\end{tabular}

*Kruskal-Wallis Test, ${ }^{*}$ Mann-Whitney $\cup$ Test, ": difference between radiographic axSpA and non-radiographic axSpA, \#\#: difference between radiographic axSpA and healthy controls, IQR 25/75: Interquartile range 25/75, BMI: Body Mass Index, BASMI: Bath Ankylosing Spondylitis Metrology Index, BASFI: Bath Ankylosing Spondylitis Functional Index, BASDAI: Bath Ankylosing Spondylitis Disease Activity Index, NA: Not Applicable, $p<0.05$

Conclusion: The results of the present study suggest that radiographic damage in axSpA may alter the physical activity levels. Every effort should be taken to increase physical activity levels in axSpA patients, especially in radiographic cases.

Acknowledgments: This project was supported by Izmir Katip Celebi University Scientific Research Projects Coordinatorship.

Disclosure of Interests: None declared

DOI: 10.1136/annrheumdis-2020-eular.4231

\section{THU0639-HPR GOUT DISEASE ACTIVITY SCORE - FURTHER EVALUATION OF CONSTRUCT AND CONVERGENT VALIDITY}

M. Zlatkovic-Svenda ${ }^{1,2}$, M. Radak-Perovic ${ }^{1} .{ }^{1}$ Institute of Rheumatology, University of Belgrade School of medicine, Belgrade, Serbia; ${ }^{2}$ Medical Faculty, University of East Sarajevo, Republika Srpska, Foca, Bosnia and Herzegovina

Background: A new instrument for measuring of disease activity in gout is a 4-variable gout disease activity score (GAS), announced in 2016 (1). GAS calculates self-reported number of attacks in the last year, patient visual analogue scale (VAS) for gout severity, serum uric acid level $(\mathrm{mg} / \mathrm{dL})$ and the number of tophi, and has shown good psychometric properties (1).

Objectives: to test GAS in patients with gout and to evaluate its construct and convergent validity

Methods: 74 patients with gout were evaluated successively, as they entered the Institute of Rheumatology in Belgrade. Construct validity was assessed by associationos between GAS and SF-36, using Spearman's rank correlation coefficients. Convergent validity was evaluated by testing the ability of the GAS score to distinguish between patients grouped by perceived disease severity, perceived general health and physicians perceived disease severity by using Kruskal-Wallis one-way analysis of variance. Computer statistic program SPSS 20.0 was used for data evaluation.

Results: For the construct validity, significant correlations were found between GAS and SF-36 subscales, specially for Body pain ( $r=-0.725)$, Physical functioning ( $r=-0.684)$ and Physical limitations $(r=-0.563)$, whereas Social functioning, Mental health, and Vitality were less affected. Correlation between GAS and Physical component scale of the SF-36 was the most prominent $(r=-0.780)$. For the convergent validity, significant differences in GAS scores were found between patients grouped by perceived general health $(p<0,01)$ and perceived disease severity $(p<0,01)$, and by doctors perceived disease severity $(p<0,01)$, demonstrating the ability of the GAS to distinguish between subgroups of patients.

Conclusion: GAS has shown good psyshometric properties in Serbian cohort of patients and has proved to be valid and reliable tool for use in clinical practice. 